\title{
AN IN VIVO MICROFABRICATED SCAFFOLD FOR TENDON REPAIR
}

\author{
A.S.G. Curtis ${ }^{1 *}$, C.D.W. Wilkinson ${ }^{2}$, J. Crossan ${ }^{3}$, C. Broadley ${ }^{1}$, H. Darmani ${ }^{1,4}$, K.K. Johal ${ }^{1,5}$, H. Jorgensen ${ }^{2,6}$ and W. \\ Monaghan ${ }^{2}$ \\ ${ }^{1}$ Centre for Cell Engineering, University of Glasgow, Glasgow G12 8QQ, UK. \\ ${ }^{2}$ Department of Electronics and Electrical Engineering, University of Glasgow,Glasgow G12 8QQ,UK. \\ ${ }^{3}$ Department of Orthopaedics, Western Infirmary, Glasgow G11 UK, \\ ${ }^{4}$ Dept. Applied Biology, Faculty of Science, Jordan University of Science and Technology, PO Box 3030, Irbid \\ 22110, Jordan. \\ ${ }^{5}$ SCP Science, 21800 Clark Graham, Baie d'Urfe Quebec Canada, \\ ${ }^{6}$ ATMU and Cancer Division, Glasgow Royal Infirmary, Glasgow G31 2BR
}

\begin{abstract}
A new type of in vivo tissue engineering system for tendon repair in situ after cut or crush of a flexor tendon is described. The system is based on the topographical reaction, alignment, migration and perhaps proliferation of tendon cells on micrometrically grooved substrates made in a biodegradable polymer. Macrophage trapping in the structure may also help to prevent inflammation. Tendon damage including crush and section injury is a fairly frequent occurrence. The conventional treatment is surgical repair, however frequently this leads, especially in hand wounds, to attachment of the tendon surface to the surrounding synovium, which is very undesirable. We present an approach based on using a biodegradable device to ensure that the healing of severed or crushed flexor tendons is aided, synovial adhesion prevented and the final result anatomically correct. The biodegradable sheath carries microgrooves fabricated into the polymer by embossing that orient and guide the cells towards each other from either side of the region of damage. After six weeks an apparently normal functional tendon is reformed.
\end{abstract}

Key Words: Tendon repair, micrometric scaffold, cell orientation, collagen reformation.

*Address for correspondence:

A.S.G. Curtis

Centre for Cell Engineering,

University of Glasgow

Glasgow G12 8QQ. U.K.

E-mail: A.curtis@bio.gla.ac.uk

\section{Introduction}

Wojciak et al. (1995) found that rat tendon explants grown in organ culture in pairs, separated by a narrow gap of 1$4 \mathrm{~mm}$ on planar glass surfaces produced a plentiful monolayered outgrowth of fibroblast-like cells without effective union of the explants. The cells grew out from the epitenon, which lies at the outer surface of the tendon. When they were grown on a grooved substrate with the grooves directed between the explants good fusion was observed in three weeks with the new tissue forming a rod-like union between the two pieces. This alignment is an example of contact guidance; see Weiss (1945) and also Curtis and Varde (1964). In other words the grooved microstructure aligned the cell migration from the cut tissue ends so that the cells migrated towards each other to form a sheet joining the two pieces of tendon. The first layer of cells appeared to align a second layer of cells that grew over the first layer, and subsequent layers similarly.

Those results suggested that we might be able to align cells emigrating from the ends of a crushed or cut tendon with a grooved substrate so that the cut ends reunite into a strong correctly structured functional tendon. Collagen might also become aligned (Wang et al., 2003), which would be an essential feature of new functional tendon. In order to do this we designed the prosthetic device shown in principle in Figure 1. A different device proposed by Ricci et al. (1991) is partially similar in having cell alignment (to polymer fibres) as a support material for tendon re-growth but their system does not supply immediate mechanical support for the tendon nor does it separate synovium and tendon. Niemeyer et al. (2004) have studied a collagen/calcium triphosphate scaffold as a candidate material for tendon repair. Calve et al. (2004a,b) obtained a tendon-like construct in the absence of a scaffold by stretching the cells between two anchor posts. Surprisingly until now there have been no publications on using contact guidance on grooves to influence tendon repair nor further in vitro observations of tenocyte behaviour on grooved materials.

We suggest that the results of our research show that our proposal achieves all the required aims for tendon repair.

The basic device in concept consists of a sheet of biodegradable polymer grooved on the face applied to the tendon and smooth on the outer surface. Ideally the device might be slid over the two ends of the tendon and 

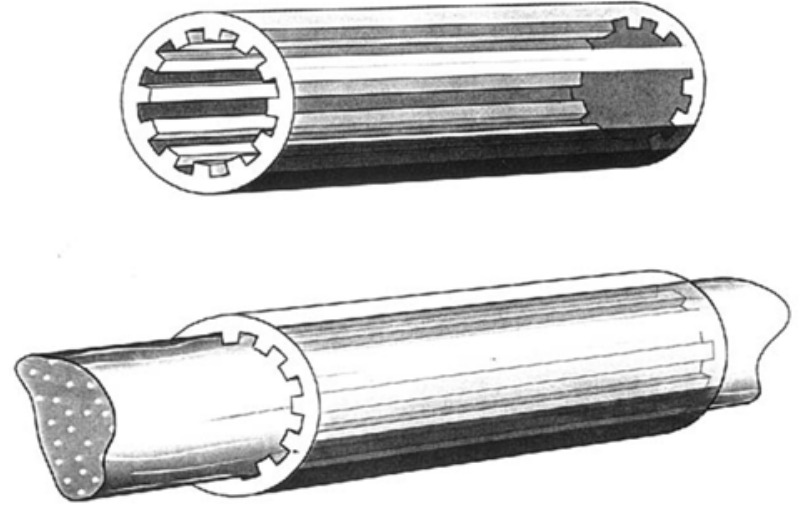

Figure 1. Schematic view of concept of the device. Top picture A tube of polydioxanone sheet (PDS) microgrooved on the inside into which (lower picture) the tendon ends are inserted. Not to scale. Groove depths and widths exaggerated for clarity.

then its ends sutured onto the two ends of the tendon. The sutured sheet should provide initial strength and alignment for the tendon. In a variant of this device for use with crush injuries where the tendon is not severed the grooved sheet is wrapped around the tendon and sutured to the tendon at either end and possibly along the free lateral edges of the sheet. Then the grooves align the cells as they move out of the tendon ends and the polydioxanone (PDS) sheet keeps the synovium from fusing with the tendon. By the time the PDS has degraded the surface of the tendon is sufficiently repaired and polarised to have a non-adherent surface facing the emerging synovial space so that tendon to synovium adhesion does not occur.

This paper describes in vivo tests of this device which is designed to prevent other problems associated with simple surgery on tendons or use of other types of scaffold, see for example Rooney et al. (1993), Newmeyer and Manske (2004), Louie et al. (1997), Leibovic and McDowell (1994) and Cao et al. (1994).

\section{Materials and Methods}

\section{Fabrication of master dies}

Fused silica plates, $100 \mathrm{~mm} \times 100 \mathrm{~mm} \times 1 \mathrm{~mm}$ thick, from (Multi-lab, Newcastle, UK) were used. The silica was cleaned by soaking in a solution of 7:1 $98 \%$ sulphuric acid: hydrogen peroxide for $5-10$ minutes at $70^{\circ} \mathrm{C}$ followed by a rinse in Reverse Osmosis (RO) water, then blown dry with filtered air. The silica was coated with S1818 photoresist (Shipley, Coventry, UK) by spinning-on at 4000 rpm for 30 seconds followed by a soft bake at $90^{\circ} \mathrm{C}$ for 30 minutes. This gave a resist thickness of $1.8 \mu \mathrm{m}$. The resist was then patterned by exposing to ultra-violet light for 10 seconds through a chrome mask patterned with the required grating pattern, using a mask aligner (HTG Inc, San Jose, CA, USA). The exposed resist was removed by immersing the sample in a solution of 1:1 Shipley Microposit developer: RO water for 65-75 seconds followed by a rinse in $\mathrm{RO}$ water and blown dry. The samples were etched in a
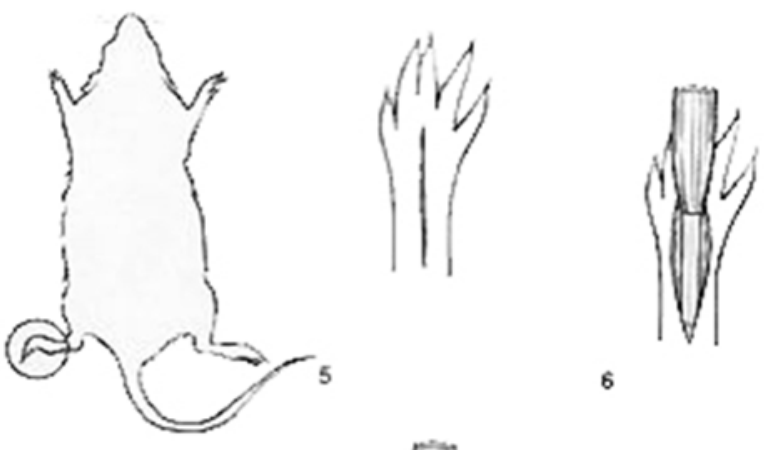

4

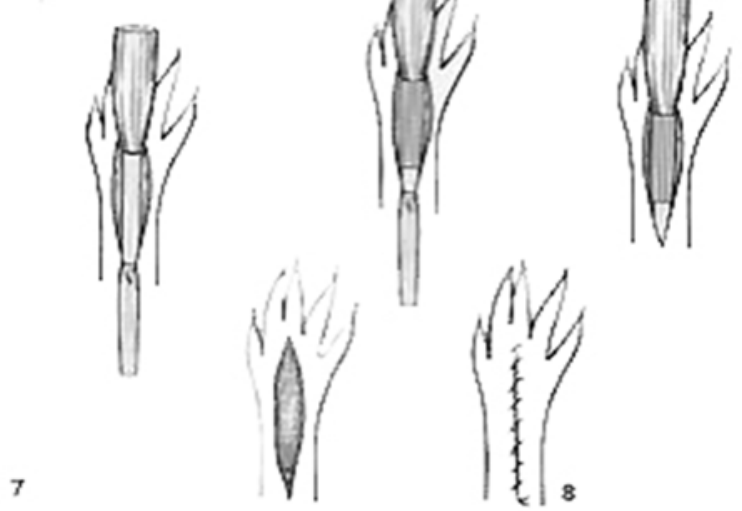

Figure 2. Schematic of the operative procedures. 1.Rat placed on its back to assist its breathing 2. Scalpel blade Size 10 used to cut along mid-palmar side of the foot. 3. Cut is opened up and using a pair of forceps the muscle is separated from the tendon. Muscle is cut at base of incision into the skin. 4. Synovial canal is exposed and tendon cut (or crushed). 5. The implant is placed under the tendon at the level of the cut and the implant is pulled by its upper surface over the tendon with its under surface moving underneath the tendon. Alternatively the implant has been made in two separate sheets and the sheets are separately worked above and below the tendon. 7 . The muscle flap is sutured back and the overlying tissue replaced. 8 The skin is sutured. Crush damage can only be treated by the sandwich technique.

Reactive Ion Etching Unit (Plasma Technology, Bristol, UK) in trifluoromethane gas. After etching the residual resist was removed, and all samples etched for an additional minute without protection to ensure uniform surface chemistry. The step heights (depth of groove), which were $5 \mu \mathrm{m}$, were measured with a Dektak 23 stylus device sensitive to $0.5 \mathrm{~nm}$ (Sloan Technology, Santa Barbara, CA, USA). Structures were either used as supplied or by reusing structures after cleaning (for method see below).

\section{Cleaning structures}

After use in embossing (see below) die structures were cleaned with the hydrogen peroxide/sulfuric acid reagent described above, rinsed well with RO water and stored in $100 \%$ ethanol until shortly before use. They were then dried by evaporation in a sterile airflow. 


\section{Polydioxanone (PDS)}

This polymer was a gift from Ethicon Ltd., Edinburgh, U.K.

Fabrication. Polydioxanone samples freshly taken from nitrogen storage were melted between flat glass platens in a hot press at a pressure of $250 \mathrm{psi}\left(175750 \mathrm{~kg} . \mathrm{m}^{-2}\right)$ and a temperature of $90^{\circ} \mathrm{C}$ to form flat sheets. These sheets were then re-melted in a hot press with one face of the press being a silica master. On cooling the master dies and the sheet were removed and the PDS grooved sheet peeled off the master. The sheets were stored dry and briefly soaked in $70 \%$ alcohol for sterilization immediately prior to implantation

Implantation of sheets in vivo. Microsurgery. Operation. The operations were carried out under Home Office Licence and local Ethical Committee approval. The general protocol of the operations is shown in Figure 2. Male Sprague-Dawley rats weighing in the range of 250-300 grams were anaesthetised using Halothane.

Under tourniquet control, a longitudinal incision was made in the planar surface of the hindpaw and extended along the line of the middle digit to expose the synovial canal. The superficial tendon was lifted together with the attached muscle to expose the deeper flexor tendon which was then severed at one end. The free tendon was carefully raised and the PDS implant (cut to fit) was inserted with the inner aspect of the membrane (either plain or longitudinal/ transverse grooves) in close proximity to the tendon. The overlying tissue consisting of muscle and superficial tendon were then allowed to fall back onto the implant. In this way, the tendon was sandwiched between two sheets of PDS. Control operations were carried out on the contralateral paw in which no PDS sheet was implanted.

Implanted subjects and controls were harvested at 3, 7, 14, 28, 42 and 73 days subsequent to implantation and the paws processed for histology. The animals were sacrificed by $\mathrm{CO}_{2}$ asphyxiation followed by cervical dislocation at various time points following completion of the operation. The tendon areas were fixed (see below) after excision.

Histology methods. Fixation and permeabilisation. 4\% Formaldehyde in phosphate buffered saline (PBS) pH 7.4 was used for routine fixation for histology with fixation times of 30 minutes followed by storage in Hepes buffered saline with $0.001 \%$ sodium azide for bulk tissue specimens if prolonged storage was expected. The fixation time for cultures and thin cellular membranes was reduced to 3 minutes. For material to be immuno-stained or rhodaminephalloidin stained the material (cell cultures or thin cellular membranes such as the epitenon) a permeabilising fixation was used, This used a 3 minute fixation in 3\% formaldehyde in phosphate buffered saline followed by 10 minutes in the same fixative with $0.5 \%$ Triton $\mathrm{X}-100$,

Histology

Tissue examination. Eosin staining and haematoxylin was used on 5-micrometre paraffin wax sections of the fixed tissue and van Gieson staining was used to reveal collagen staining. Mouse antibodies at 1/200 titre against ED1 and ED2 antigens (Serotec, Oxford, UK (www.serotec.co.uk)) (MCA341 and MCA342) were reacted with the dewaxed sections followed after washing in saline by a polyclonal goat antimouse antibody (1/400) conjugated to alkaline phosphatase to produce Fast Red staining of reactive cells, These two antibodies were used to reveal inactive (newly recruited ED1+) and activated (mature) macrophages $(\mathrm{ED} 2+)$. All antibody reactions were done at $4^{\circ} \mathrm{C}$ in saline at $\mathrm{pH} 7.6, \mathrm{I}=0.15$, Rhodamine-phalloidin staining was used to reveal actin orientation in isolated epitenon. The rhodamine phalloidin stock used had a concentration of $200 \mathrm{U}$ in $1 \mathrm{ml}$ methanol) and this was used at 1:50 in tris saline $\mathrm{pH} 7.6, \mathrm{I}=0.15$, for 1 hour on the fixed permeabilised samples at $4^{\circ} \mathrm{C}$. After this staining the samples were washed in saline four times.

\section{Staining of trapped macrophages and thin epitenon mounts.}

At 1-3 days after implantation some of the operated tendons were removed immediately after the animals had been sacrificed and the PDS membranes stained with $1 \%$ aqueous methylene blue. Macrophages, activated or otherwise, were identified by their positive reaction with ED1 and ED2 antibodies. The 1 week regenerate was examined by dissection of the very thin membrane from the surface of the PDS after a brief fixation in $3 \%$ buffered formaldehyde with 1\% triton X100 to permeabilise the cells. The sections were washed in Trisbuffered saline (TBS), total ionic strength $0.15, \mathrm{pH} 7.6$ and treated with dilute $1 \%$ bovine serum albumin (Sigma, St. Louis, MO, USA) in PBS pH 7.6 for $15 \mathrm{~min}$. The slides were then drained and incubated with mouse antirat macrophage clone ED1 and ED2 (Serotec) (prepared in TBS with bovine serum albumin $1 \%$ ) for $1 \mathrm{~h}$ at room temperature $25^{\circ} \mathrm{C}$ at a dilution of 1:500. After washing three times in TBS for $5 \mathrm{~min}$, the slides were treated with biotin-conjugated secondary goat antimouse antibody for $45 \mathrm{~min}$ at room temperature. Subsequent to three further 5 min washes in TBS, the sections were incubated with alkaline phosphate-conjugated extravidin (Sigma) at a dilution of 1:1000 for $30 \mathrm{~min}$. The sections were washed three times in TBS and then visualised using Fast 5-bromo-4-chloro-3 indolylphosphate/nitro-blue tetrazolium substrate (Sigma) $0.2 \%$. Note the different procedures used on this material as compared to the whole fixed tissue which had been sectioned.

The epitenon membrane was stained with rhodamine conjugated phalloidin and fluorescence microscopy used to reveal any actin orientation in the membrane. Macrophages and leucocytes adhering to grooved PDS surfaces were revealed with $1 \%$ methylene blue in buffered saline.

\section{Polarisation microscopy: collagen staining}

A proportion of frozen sections from the repaired tendon were examined by polarisation microscopy (Polscope CRI, Waltham, MA USA) to view collagen fibres. Using this method of microscopy collagen and the remains of the PDS were seen as the only brightly birefringent objects. Van Gieson staining also suggested that collagen was present through the length of the repaired area. 


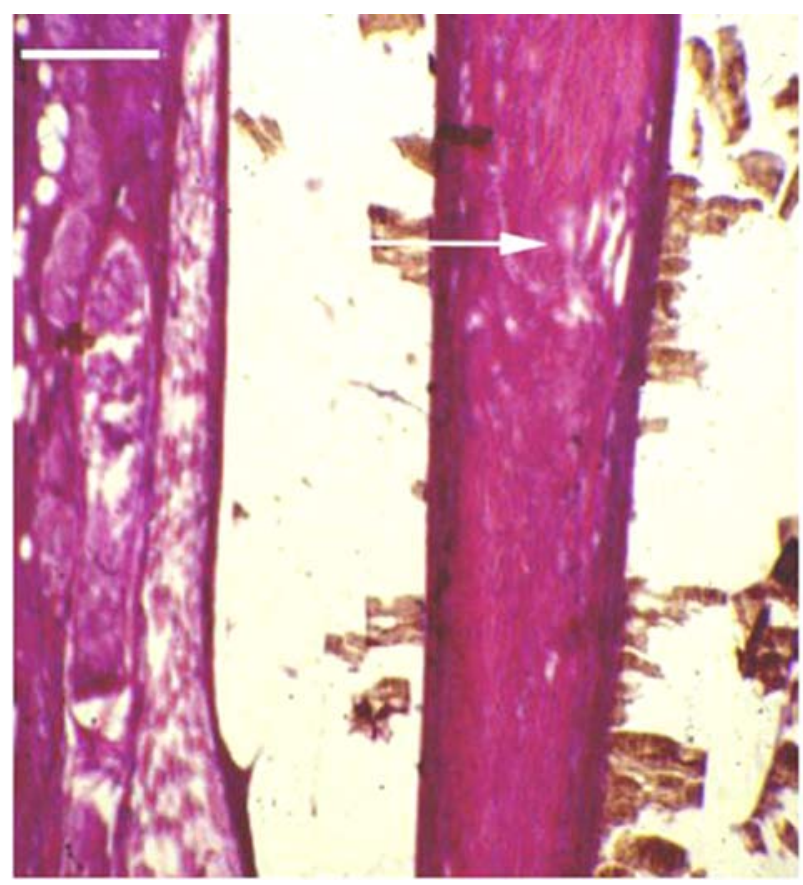

Figure 3. Tendon repair at 4 weeks. A typical wellrepaired tendon in longitudinal section after cutting and implantation of a PDS grooved device as shown in figure 2. Traces of degraded PDS can be seen in the synovial spaces. Synovium on left. Some voids in region of crush indicated by arrow. Epitenon complete, lining outside of tendon. Synovial spaces on either side of tendon. Haematoxylin and eosin staining. Scale bar 80 micron.

\section{Results}

\section{Course of repair}

In all of the experiments the wounds healed by primary intention and there was no indication of lameness in any of the animals. The animals had normal gait. When the 14 day and 28 day healed tendons were inspected it was found that they had healed by continuity of scar tissue, which remodelled over 2-3 weeks into normal tendon, that they were non-adherent to the PDS membrane and that gentle traction on the tendon produced unimpaired flexion of the digit. The final results of repair are shown in Figures, 3, 4 and 5, Controls are shown in Figures $6 \mathrm{a}$ and $6 \mathrm{~b}$.

At 3 days after operation a very thin layer of oriented epitenon cells was found covering the inside of the PDS implant (Fig. 7). Just outside this layer of epitenon cells macrophages have accumulated on the ridges of the PDS facing the tendon, In control experiments done with smooth surfaced PDS there is no cell orientation in this first layer of cells and macrophages (rounded cells) have no special location, 4 weeks post implantation (Fig. 8).

The samples harvested at earlier time intervals after operation were mainly used for histological examination to detail the course of repair and were from animals with the grooved PDS implanted around the tendon or animals in which no PDS implant had been made. A summary of the results is given in Table $1 \mathrm{a}$ and $1 \mathrm{~b}$.

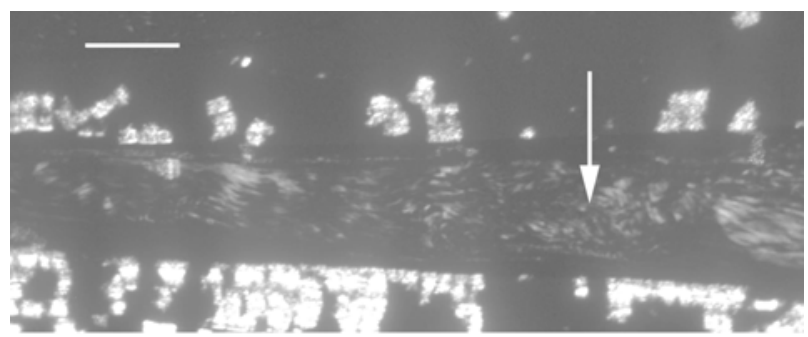

Figure 4. Polarisation microscope image of tendon (longitudinal section) and degrading polydioxanone sheath showing break-up of the degrading polymer (white fragments), Collagen in tendon clearly shown as grey-white areas, other areas in tendon which are dark are presumably cells or voids. Region of sectioning shown by arrow. Scale bar $60 \mu \mathrm{m}$.
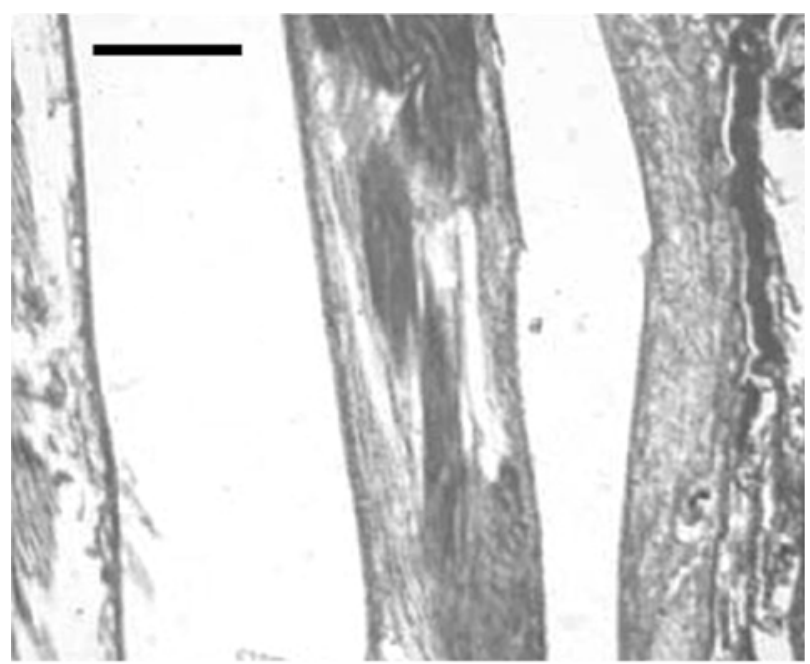

Figure 5. Repair of a crushed tendon. Grooved PDS implant used. Four weeks post operation. Tendon running vertically in centre. Van Gieson stain. Scale bar $100 \mu \mathrm{m}$.

A remarkable feature of the presence of the device is the trapping of macrophages on the ridge surface of the PDS (Fig. 9). This may sequester them from further phagocytic activity or alternatively provide a source of macrophage/monocyte cytokines, see Darmani et al (2004a,b). Those tendons examined after 4 weeks of repair have a very low level of included macrophages which suggests that inflammation is minimal.

\section{Collagen reformation}

Collagen reformation and alignment in the repaired tendons is normal in those animals with cut or crushed tendon and grooved PDS implants arranged with the grooves running longitudinally.. Collagen orientation develops in these animals with the repaired tendon running through the region of injury. Polarisation microscopy images of a section are shown in Figures 10a and 10b. When the grooved implant is oriented at 90 degrees to the long axis of the tendon with the grooves running transversely like hoops collagen formation follows the grooves and is incorrect by 90 degree. Animals with no implant or smooth PDS implants had poorly organised collagen. 


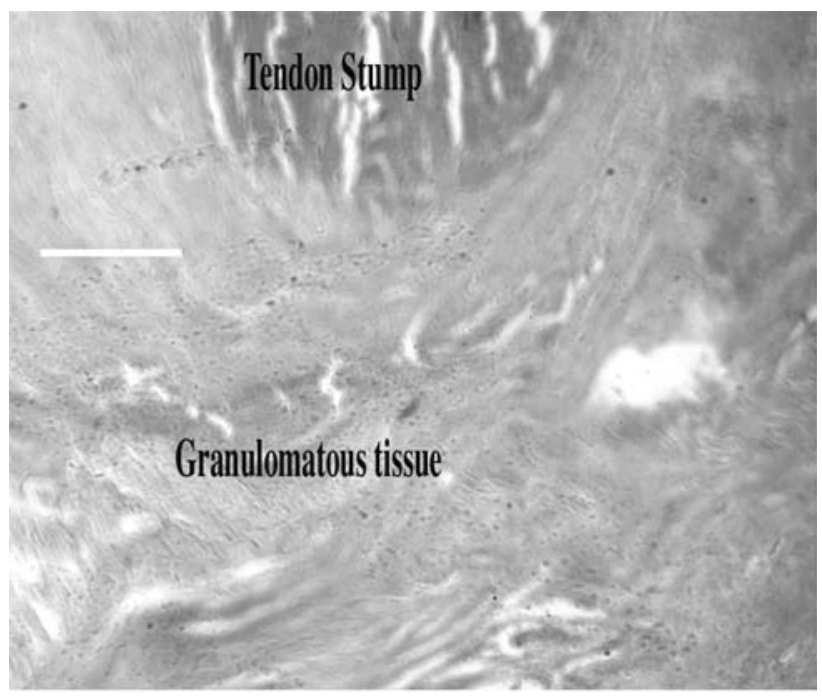

Figure 6a. Control 4 weeks, no device inserted. Longitudinal section in region of cut. The missing synovial space found in all cases is presumably due to tendon or synovial trauma during the operation. Note tendon cut end remains as a stump. Scale bar $100 \mu \mathrm{m}$..

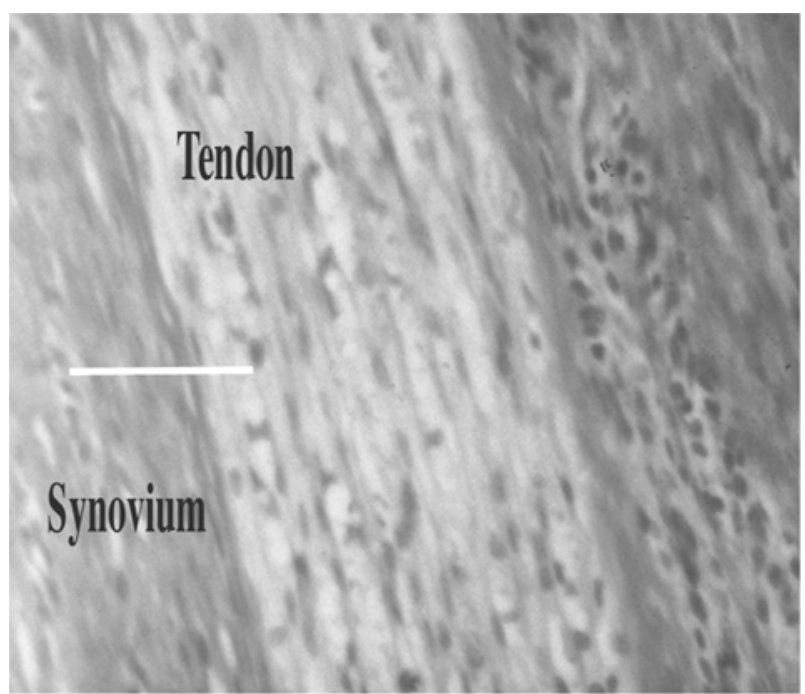

Figure 6b. Control 4 weeks, no device inserted Longitudinal section remote to cut. The missing synovial space found in all cases is presumably due to tendon or synovial trauma during the operation. Scale bar $80 \mu \mathrm{m}$.

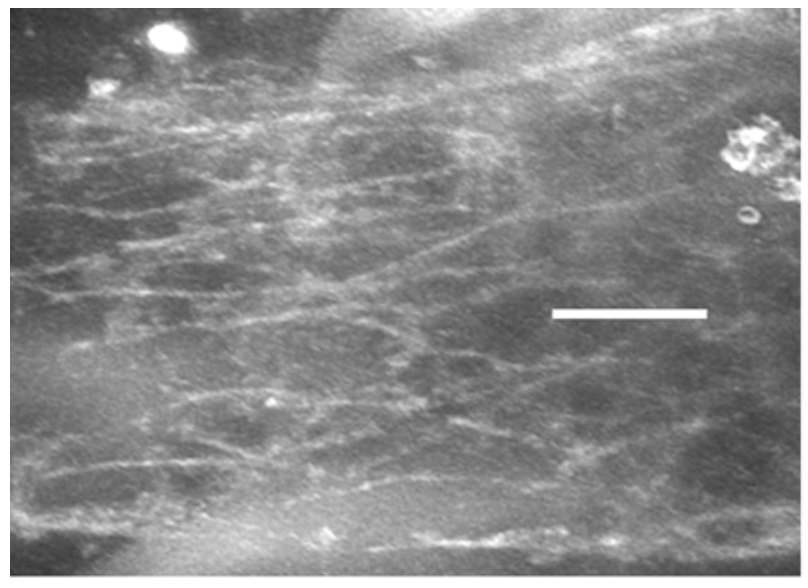

Figure 7. Oriented epitenon at 3 days on grooved PDS. Stained with fluorescent phalloidin Groove orientation was along the length of the tendon. Note cells are elongated in the same direction. Cells outlined by Factin staining at cell boundaries. Scale bar $20 \mu \mathrm{m}$.

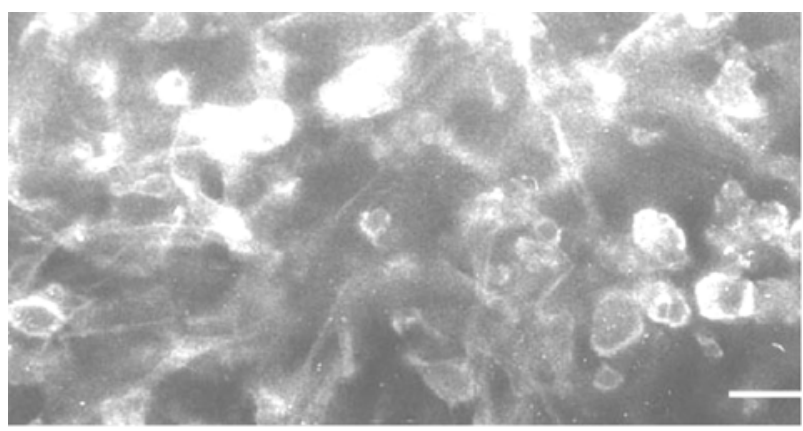

Figure 8. Cells in the layer next to the grooved side of the PDS after 3 days. Plain ungrooved membrane. Note the macrophages (round) and the unoriented fibroblastlike cells. Staining fluorescent phalloidin. Scale bar 20 $\mu \mathrm{m}$.
Table 1a 28 days post-operation

$\begin{array}{lcccc} & \begin{array}{c}\text { Reunion } \\ \text { failed }\end{array} & \begin{array}{c}\text { Synovial } \\ \text { space } \\ \text { missing }\end{array} & \begin{array}{c}\text { Normal } \\ \text { tendon } \\ \text { histology }\end{array} & \text { Histology } \\ \begin{array}{l}\text { Sham operated } \\ \text { No device, tendon cut }\end{array} & 10 / 10 & 10 / 10 & 0 / 10 & \text { Poor } \\ \text { PDS implant no grooves } & 1 / 6 & 3 / 6 & 0 / 6 & \begin{array}{c}\text { Tendon } \\ \text { poorly } \\ \text { oriented } \\ \text { PDS implant grooved }\end{array} \\ & 0 / 6 & 1 / 6 & 5 / 6 & \text { Good }\end{array}$

Table 1 b 42 days post operation

$\begin{array}{cccc} & \text { Synovial } & \text { Normal } & \\ \text { Reunion } & \text { space } & \text { tendon } & \\ \text { failed } & \text { missing } & \text { histology } & \text { Histology }\end{array}$

$\begin{array}{lcccc}\begin{array}{l}\text { Sham operated } \\ \text { No device, tendon cut }\end{array} & 7 / 7 & 7 / 7 & 0 / 7 & \text { Poor } \\ \text { PDS implant no grooves } & 1 / 6 & 2 / 6 & 0 / 6 & \begin{array}{l}\text { Tendon } \\ \text { poorly } \\ \text { oriented }\end{array}\end{array}$

PDS implant grooved $\quad 0 / 6 \quad 0 / 6 \quad 6 / 6$

Good

The remarks under 'Histology' in Tables $1 \mathrm{a}$ and $1 \mathrm{~b}$ have the following meaning : 'Poor' much granulomatous tissue, many capillaries, and synovia not clearly separate from tendon tissue. "Tendon poorly oriented' means that repaired tendon tends to retain signs of cut or crush injury and collagen fibres not continuous across injury area. 


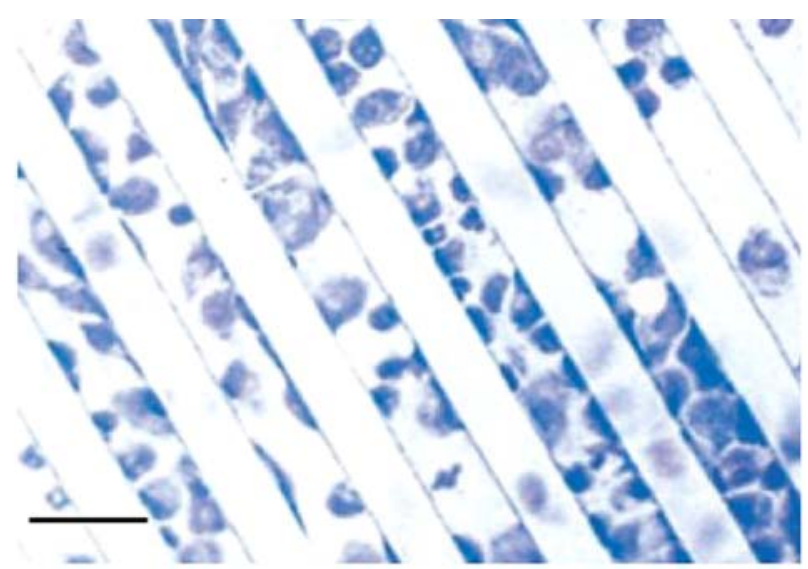

Figure 9. Macrophage trapping on ridges. A few leucocytes can be seen in the grooves palely stained. 3 days after operation. Methylene Blue 1\% staining. Scale bar $12.5 \mu \mathrm{m}$.

In Figure 10b (a transverse section across the tendon) collagen orientation is 90 degrees out of normal alignment when the grooves run at 90 degrees to the normal orientation, which is evidence for the major effect contact guidance may play in collagen orientation.

\section{Discussion}

Implantation of grooved material in the form of a sheath joining the cut or crushed/tendon ends leads to successful tendon repair. This was not found in the controls without implanted PDS. Implantation of the ungrooved sheet produces less well-oriented repair tissue than grooved membrane.

The reason why we suggest that repair of a normal tendon has taken place when a microgrooved PDS device was used to repair crushed or cut tendons, are 1.The tendon had no adhesions to the synovium or anywhere near the regions of crush or cut. 2. The synovial space was normal in appearance. 3. The animals had normal gaits. 4. The polydioxanone device had been fully degraded by the end time points. 5. Vascularisation of the tendon was slight which is normal. 6. Collagen alignment in the tendon was normal. 7. There was no noticeable accumulation of inflammatory cells in the tendon or surrounding tissues.

Reconstruction of events of repair was as follows: 1 . One to three days - The sutured membrane kept the synovium and tendon apart and provided mechanical strength for the tendon. Macrophages accumulated on the ridges, presumably trapped there, but were not elongated. The epitenon from the undamaged ends grew into the area of damage as a layer guided by the grooved structures. Cells in this initial layer were elongate in the direction of the grooves (Fig. 7) but were not in the controls (Fig. 8). 2 . Four days to 2 weeks - Further cell migration occurred on the inside of the epitenon and was guided by the oriented epitenon cells. Towards the end of this period collagen remodelling may start and appreciably more collagen was found in the repair area than earlier. Two to 4 weeks - The

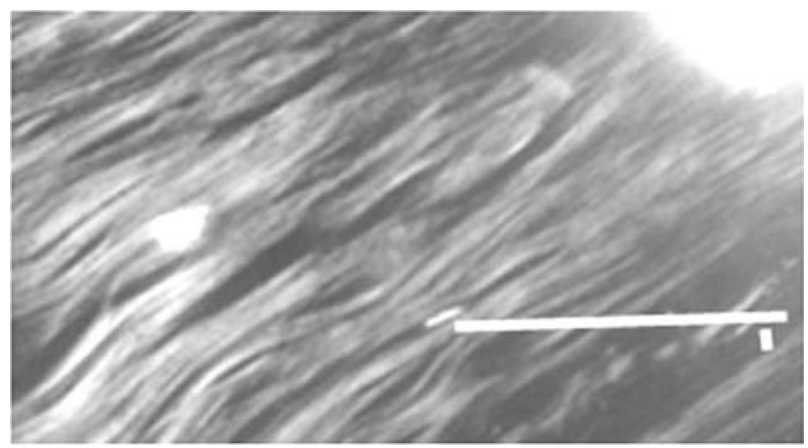

Figure 10a. Collagen formed after repair. Grooves aligned to long axis of tendon. Collagen appears bright in this polarising light picture. Very bright objects are fragments of PDS. Scale bar $30 \mu \mathrm{m}$.

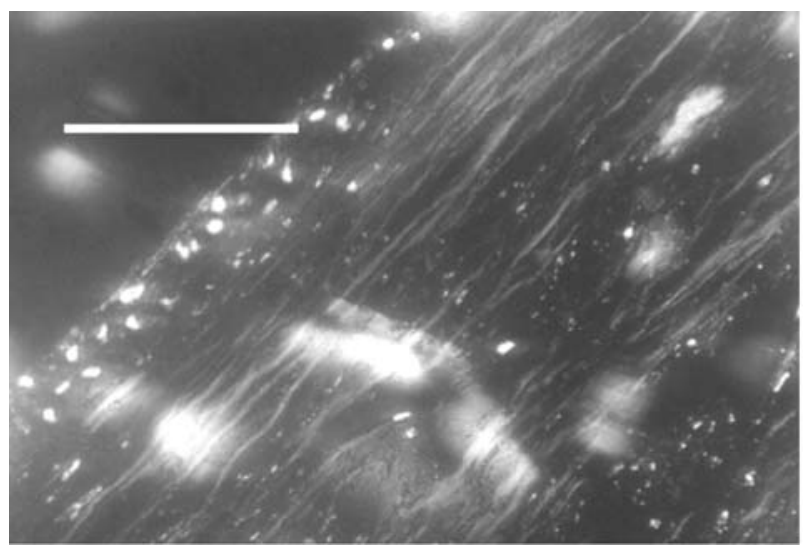

Figure 10b. Collagen formed after repair. Grooves aligned at 90 degrees to long axis of tendon. Collagen appears bright in this polarised light picture. Other bright objects are fragments of PDS. Scale bar $35 \mu \mathrm{m}$.

reformation of a collagen-rich endotenon proceeded and degradation of the PDS presumably proceeded but was not obvious histologically since the PDS membrane did not break up at this stage (Fig. 8). Four to 8 weeks Remodelling completed and the PDS started to break up and degrade (Figs. 3 and 4).

The system was effective in producing repair of a cut or crushed flexor tendon. This repair was histologically correct and which as judged by the behaviour of the animals allowed normal gait.

The success of this device for tendon repair lies we suggest in three elements of the system. First, the PDS sheath keeps the synovia and tendon apart until late in the repair process so that they do not adhere. Second, the grooves align the tendon cells correctly and possibly accelerate the cell migration into the wound area. Third, the PDS degrades at approximately the correct time to free the repaired tendon. An additional component may arise from the macrophage trapping on the ridges in the device. The probable reason for this trapping may lie in the very adhesive nature of 'convex' sharp discontinuities on the grooved PDS. The question arises as to whether this trapping reduces macrophage migration into the tendon and thus diminishes inflammation or do macrophage products such as cytokines actually aid tendon repair. 
This system appears to operate by the first layer of cells aligning the second and then the second the third and so on. This suggests that there is an alternative approach to tissue engineering rather than the conventional scaffold approach which provides the complete system in three dimensions. In the tendon system we describe the template system is for one layer of cells in the first place and the cells then copy it and impose it on subsequent layers. This rather resembles the system for corneal tissue engineering described by Nishida et al. (2004) where the tissue is built up layer on layer by adding sheets one cell layer thick at a time. In addition the system we described allows tension to be applied externally as a result of suturing and internally as a result of cellular contraction. It is relevant that Andrick et al. (2004) and also Calve et al. (2004a) provide evidence that application of mechanical tension is important for tendon repair.

It should also be noted that the degradation route for the PDS is not one proceeding by surface erosion, if it were the guiding groove structure might be lost early in the process of healing. The erosion rate was faster than expected from results with PDS sutures and this might be due either to the form of the PDS or to the re-melting of the polymer. It should be noted that the tendon is a poorly vascularised tissue so that the repair system may not require good vascular supply. Thus this system of repair may be limited to a few tissues but gives a very effective repair for the tendon system.

This method has clinical potential because it reduces inflammation, provides correct histology, obviates inappropriate adhesions and possibly gives immediate support to the animal by providing a strong polymer splint around the tendon.

\section{Conclusion}

This paper describes a method for producing effective repair of severed or crushed flexor tendons by implanting a polydioxanone sheath around the region of damage. The sheath carries microgrooves fabricated into the polymer by embossing that orient and guide the cells towards each other from either side of the region of damage. The sheath is biodegradable and prevents the tendon cells from adhering to the synovia. After six weeks an apparently normal functional tendon is reformed.

\section{Acknowledgements}

We thank Dr Grant Robertson, formerly of Ethicon, for assistance with procedures and a Link Collaborative Agreement for funding support. We also thank Dr George Marshall, Sarah McLellan and James Reilly for histology service and the Animal Services Division Glasgow University for animal care. There is no conflict of interest nor any persisting commercial support since the Link Initiative and Ethicon have relinquished the rights to the University of Glasgow.

\section{References}

Andrick JJ, Calve S, Arruda EM, Baar K (2004) Uniaxial stretch increases ERK phosphorylation and collagen synthesis in engineered tendons. FASEB J 18: A759-A760.

Calve S, Baar K, Dennis RG, Arruda EM (2004a). Morphological and mechanical characterization of selfassembling tendon constructs. FASEB J, 18: A760-A760.

Calve S, Dennis RG, Kosnik PE, Baar K, Grosh K, Arruda EM (2004b) Engineering of functional tendon. Tissue Engineering 10: 755-761.

Cao Y, Vacanti JP, Ma X, Paige KT, Upton J, Chowanski Z, Schloo B, Langer R, Vacanti CA (1994) Generation of neo-tendon using synthetic-polymers seeded with tenocytes, Transplantation Proc 26: 3390-3392.

Curtis ASG, Varde M (1964) Control of cell behaviour.topological factors. J Natl Cancer Inst 33: 15-26.

Darmani H, Crossan J, McLellan SD, Meek D, Curtis A (2004a).Expression of nitric oxide synthase and transforming growth factor-beta in crush-injured tendon and synovium. Mediators Inflammation 13: 299-305.

Darmani H, Crossan JC, Curtis A (2004b). Single dose of inducible nitric oxide synthase inhibitor induces prolonged inflammatory cell accumulation and fibrosis around injured tendon and synovium. Mediators Inflammation 13: 157-164.

Leibovic SJ, McDowell CL (1994) Repair and healing of flexor tendons of the hand. Current Opin Orthop 5: 8186.

Louie LK, Yannas IV, Spector M (1997) Healing of tendon defects implanted with a porous collagen-GAG Matrix: Histological evaluation, Tissue Engineering 3: 187195.

Newmeyer WL, Manske PR (2004) No man's land revisited: The primary flexor tendon repair controversy, $\mathrm{J}$ Hand Surgery-American Volume 29A: 1-5.

Niemeyer P, Krause U, Fellenberg J, Kasten P, Seckinger A, Ho AD, Simank HG (2004) Evaluation of mineralized collagen and alpha-tricalcium phosphate as scaffolds for tissue engineering of bone using human mesenchymal stem cells. Cells Tissues Organs 177: 6878.

Nishida K, Yamato M, Hayashida Y, Watanabe K, Maeda N, Watanabe H, Yamamoto K, Nagai S, Kikuchi A, Tano Y, Okano T (2004) Functional bioengineered corneal epithelial sheet grafts from corneal stem cells expanded ex vivo on a temperature- responsive cell culture surface. Transplantation 77: 379-385.

Ricci JL, Gona AG, Alexander H (1991) In vitro tendon cell-growth rates on a synthetic fiber scaffold material and on standard culture plates. J. Biomed Mater Res 25: 651666.

Rooney P, Walker D, Grant ME, McClure J (1993) Cartilage and bone formation in repairing Achilles tendons within diffusion chambers: Evidence for tendon-cartilage and cartilage-bone conversion in vivo. J Pathol 169:375381 .

Wang JHC, Jia FY, Gilbert TW, Woo SLY (2003) Cell orientation determines the alignment of cell-produced collagenous matrix. J Biomechanics 36: 97-102. 
Weiss PS (1945) Experiments on cell and axon orientation in vitro: the role of colloidal exudates in tissue organization. J Exp Zool 100: 353-386.

Wojciak B, Crossan J, Curtis ASG, Wilkinson CDW (1995) Grooved substrata facilitate in vitro healing of completely divided flexor tendons, J. Mater Sci: Mater in Med 6:266-271.

\section{Discussion with Reviewers}

Reviewer II: Did the authors look at paw flexion and movement to check on the mechanical strength of the connections and repair of the rats before sacrifice? What criteria were used to stop investigation with the rats with non-healing tendons?

Authors: The animals were splinted for two weeks and then the splints removed to see if gait and other behaviour and wound repair was normal. When this was not the case, veterinary advice was obtained as to whether to sacrifice the animal or use analgesia. The experimental animals had all recovered normal gait by two weeks.

Reviewer III: You suggest that the trapped macrophages might provide a source of released cytokines and that these might be beneficial. However, not all macrophage cytokine products are beneficial and they can also release harmful superoxides.

Authors: Yes this is correct. We recognise both possibilities. (Darmani et al., 2004,a,b.) in two other recent papers.

Reviewer III: Did you observe any fusion of the trapped macrophages into foreign body giant cells?

Authors: No giant cells were seen.

Reviewer III: In your final paragraph you mention that the tendon is poorly vascularised, which is correct. However, there is considerable evidence of the involvement synovial fluid in tendon repair. Would it be useful to make the PDS sheath permeable to allow the synovial fluid to permeate the tendon compartment, thus aiding the regeneration? Also, as hyaluronic acid is used in the synovial fluid as a lubricating agent, possibly this could be a beneficial bio-coating for the outer, non-grooved area of the sheath.

Authors: This could be a good idea as might application of the synovial fluid surfactant. 